\title{
Chapter 9 \\ LEAP-2017 Simulation Exercise: \\ Calibration of Constitutive Models and Simulation of the Element Tests
}

\author{
Majid T. Manzari, Mohamed El Ghoraiby, Mourad Zeghal, \\ Bruce L. Kutter, Pedro Arduino, Andres R. Barrero, Emilio Bilotta, \\ Long Chen, Renren Chen, Anna Chiaradonna, Ahmed Elgamal, \\ Gianluca Fasano, Kiyoshi Fukutake, William Fuentes, Alborz Ghofrani, \\ Koji Ichii, Takatoshi Kiriyama, Carlos Lascarro, Vicente Mercado, \\ Jack Montgomery, Osamu Ozutsumi, Zhijian Qiu, Mahdi Taiebat, \\ Thaleia Travasarou, Dimitra Tsiaousi, Kyohei Ueda, Jose Ugalde, \\ Toma Wada, Rui Wang, Ming Yang, Jian-Min Zhang, \\ and Katerina Ziotopoulou
}

\footnotetext{
M. T. Manzari ( $\square)$

Department of Civil and Environmental Engineering, George Washington University,

Washington, DC, USA

e-mail: manzari@gwu.edu

M. El Ghoraiby

The George Washington University, Washington, DC, USA

M. Zeghal

Department of Civil and Environmental Engineering, Rensselaer Polytechnic Institute, Troy, NY, USA

B. L. Kutter

Department of Civil and Environmental Engineering, University of California, Davis, CA, USA

P. Arduino · L. Chen · A. Ghofrani

Department of Civil and Environmental Engineering, University of Washington, Seattle, WA, USA
}
A. R. Barrero · M. Taiebat · M. Yang
Department of Civil Engineering, University of British Columbia, Vancouver, BC, Canada
E. Bilotta $\cdot$ A. Chiaradonna $\cdot$ G. Fasano
Department of Civil, Architectural and Environmental Engineering, University of Napoli Federico II, Naples, Italy
R. Chen
Department of Hydraulic Engineering, Tsinghua University, Beijing, China

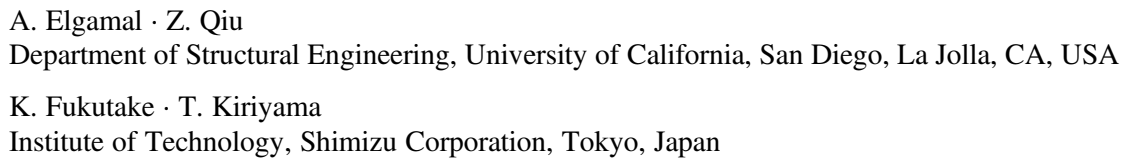




\begin{abstract}
This paper presents a summary of the element test simulations (calibration simulations) submitted by 11 numerical simulation (prediction) teams that participated in the LEAP-2017 prediction exercise. A significant number of monotonic and cyclic triaxial (Vasko, An investigation into the behavior of Ottawa sand through monotonic and cyclic shear tests. Masters Thesis, The George Washington University, 2015; Vasko et al., LEAP-GWU-2015 Laboratory Tests. DesignSafe-CI, Dataset, 2018; El Ghoraiby et al., LEAP 2017: Soil characterization and element tests for Ottawa F65 sand. The George Washington University, Washington, DC, 2017; El Ghoraiby et al., LEAP-2017 GWU Laboratory Tests. DesignSafe-CI, Dataset, 2018; El Ghoraiby et al., Physical and mechanical properties of Ottawa F65 Sand. In B. Kutter et al. (Eds.), Model tests and numerical simulations of liquefaction and lateral spreading: LEAP-UCD-2017. New York: Springer, 2019) and direct simple shear tests (Bastidas, Ottawa F-65 Sand Characterization. PhD Dissertation, University of California, Davis, 2016) are available for Ottawa F-65 sand. The focus of this element test simulation exercise is to assess the performance of the constitutive models used by participating team in simulating the results of undrained stress-controlled cyclic triaxial tests on Ottawa F-65 sand for three different void ratios (El Ghoraiby et al., LEAP 2017: Soil characterization and element tests for Ottawa F65 sand. The George Washington University, Washington, DC, 2017; El Ghoraiby et al., LEAP-2017 GWU Laboratory Tests. DesignSafe-CI, Dataset, 2018; El Ghoraiby et al., Physical and mechanical properties of Ottawa F65 Sand. In B. Kutter et al. (Eds.), Model tests and numerical simulations of liquefaction and lateral spreading: LEAP-UCD-2017. New York: Springer, 2019). The simulated stress paths, stress-
\end{abstract}

\author{
W. Fuentes \\ Universidad del Norte, Barranquilla, Colombia \\ K. Ichii \\ Faculty of Societal Safety Science, Kansai University (Formerly at Hiroshima University), \\ Osaka, Japan \\ C. Lascarro · V. Mercado \\ University of British Columbia, Vancouver, BC, Canada \\ J. Montgomery \\ Department of Civil Engineering, Auburn University, Auburn, AL, USA \\ O. Ozutsumi \\ Meisosha Corporation, Tokyo, Japan \\ T. Travasarou $\cdot$ D. Tsiaousi $\cdot$ J. Ugalde \\ Fugro, Walnut Creek, CA, USA \\ K. Ueda \\ Disaster Prevention Research Institute, Kyoto University, Kyoto, Japan \\ T. Wada \\ Department of Civil and Earth Resources Engineering, Kyoto University, Kyoto, Japan \\ R. Wang · J.-M. Zhang \\ Department of Hydraulic Engineering, Tsinghua University, Beijing, China \\ K. Ziotopoulou \\ Department of Civil and Environmental Engineering, University of California, Davis, CA, USA
}


strain responses, and liquefaction strength curves show that majority of the models used in this exercise are able to provide a reasonably good match to liquefaction strength curves for the highest void ratio $(0.585)$ but the differences between the simulations and experiments become larger for the lower void ratios (0.542 and 0.515).

\subsection{Introduction}

The LEAP-2017 project involved 11 numerical simulation teams from different academic institutions and geotechnical engineering firms across the world that participated in modeling of a selection of the performed centrifuge experiments. The simulation exercise consisted of constitutive model calibration, Type-B prediction, and Type-C prediction. This paper presents a summary of the results of the first phase (i.e., calibration) of this exercise. The main purpose of this phase was to provide the numerical simulation teams an opportunity to calibrate the constitutive models (that would be used in Type-B simulations) against the results of monotonic and cyclic triaxial tests conducted on Ottawa F-65 sand during the course of LEAP-2015 and LEAP-2017 projects. Moreover, the calibration phase would allow for a detailed documentation of the model performance against laboratory element tests before the same model is used in numerical simulations of LEAP-2017 centrifuge tests.

In preparation of the (calibration of constitutive models) phase, the following steps were followed:

- The George Washington University team performed a large number of laboratory tests (80 tests in total) on the selected soil (Ottawa F-65 sand) to obtain:

- Basic properties of the soil (particle size distribution, specific gravity, hydraulic conductivity)

- Stress-strain response and liquefaction strength of the soil using stresscontrolled cyclic triaxial tests

- A detailed report on these experimental results (El Ghoraiby et al. 2017, 2018, 2019) was prepared and provided to the participating teams in the simulation exercise.

- Additional soil characterization and element tests data (monotonic and cyclic strain-controlled triaxial tests) produced during the LEAP (Vasko 2015; Vasko et al. 2018) were also provided to the simulation teams through "datacenterhub."

- In addition to the above data, the monotonic and cyclic direct simple shear tests performed by Bastidas (2016) were also made available through "datacenterhub" (Bastidas et al. 2017).

- The laboratory test data were provided on March 14, 2017, to 20 simulation teams from around the world (9 USA, 5 Japan, 1 China, 1 Germany, 1 Italy, 1 Canada, 1 Colombia, 1 New Zealand) that originally expressed interest in participating in the LEAP-2017 simulation exercise.

- The deadline for submission of the calibration simulations was set as June 1, 2017. 
- Twelve teams submitted their calibration simulations by or near the deadline. The participating simulation teams submitted 17 different numerical simulations.

The element test results reported in El Ghoraiby et al. $(2017,2018,2019)$ are the target of the calibration simulations. Each numerical simulation team submitted a detailed report discussing the process followed in the calibration of their selected constitutive model. The reports provided a description of the constitutive model used by the predictors as well as the method used for calibration. A brief summary of these reports is presented in the papers by each numerical simulation teams in the proceedings of LEAP-UCD-2017 workshop. A summary of the element tests simulations and results is presented in the following sections.

Here it is important to note that triaxial tests were chosen in the present study due to availability of equipment and based on the fact that the boundary conditions are more accurately defined in triaxial tests than in simple shear tests. Majority of the element tests were performed using an initial effective stress of about $100 \mathrm{kPa}$. This effective stress is larger than the vertical effective stress at mid-depth of the centrifuge model which was about $20 \mathrm{kPa}$ in the middle of the deposit. The density of the sand specimens in the triaxial tests corresponded to relative densities of $71.5 \%, 87.5 \%$, and $97.5 \%$. The relative densities of the soil specimens in the centrifuge tests ranged from about $40 \%$ to $80 \%$. In the following LEAP projects, a new series of direct simple shear and hollow cylinder torsional shear tests are planned so that constitutive models can be calibrated at conditions closer to that in the centrifuge tests.

\subsection{The Numerical Simulation Teams}

The numerical simulation teams that submitted their calibration reports and participated in the Type-B simulation exercise are listed in Table 9.1.

Table 9.1 Numerical simulation teams

\begin{tabular}{|c|c|c|c|}
\hline No & Numerical simulation team & Constitutive model & $\begin{array}{l}\text { Analysis } \\
\text { platform }\end{array}$ \\
\hline 1 & Tsinghua University & Tsinghua Constitutive Model & OpenSEES \\
\hline 2 & Meisosha Corporation & Cocktail Glass Model & FLIP Rose \\
\hline 3 & Shimizu Corporation & Bowl Model & HiPER \\
\hline 4 & University of Napoli Federico II & Hypoplastic Model & Plaxis \\
\hline 5 & UC Davis-Auburn University & PM4Sand Model & Flac-2D \\
\hline 6 & University of Washington & $\begin{array}{l}\text { Manzari-Dafalias Model/PM4Sand } \\
\text { Model }\end{array}$ & OpenSEES \\
\hline 7 & Kyoto University & Cocktail Glass Model & FLIP TULIP \\
\hline 8 & Universidad del Norte & ISA-Hypoplasticity Model & ABAQUS \\
\hline 9 & University of British Columbia & SANISand & FLAC-3D \\
\hline 10 & $\begin{array}{l}\text { University of California, } \\
\text { San Diego }\end{array}$ & PDMY & OpenSEES \\
\hline 11 & Fugro West & PM4Sand Model/UBCSAND & FLAC-2D \\
\hline 12 & Hiroshima (Kansai) University & Cocktail Glass Model & FLIP Rose \\
\hline
\end{tabular}



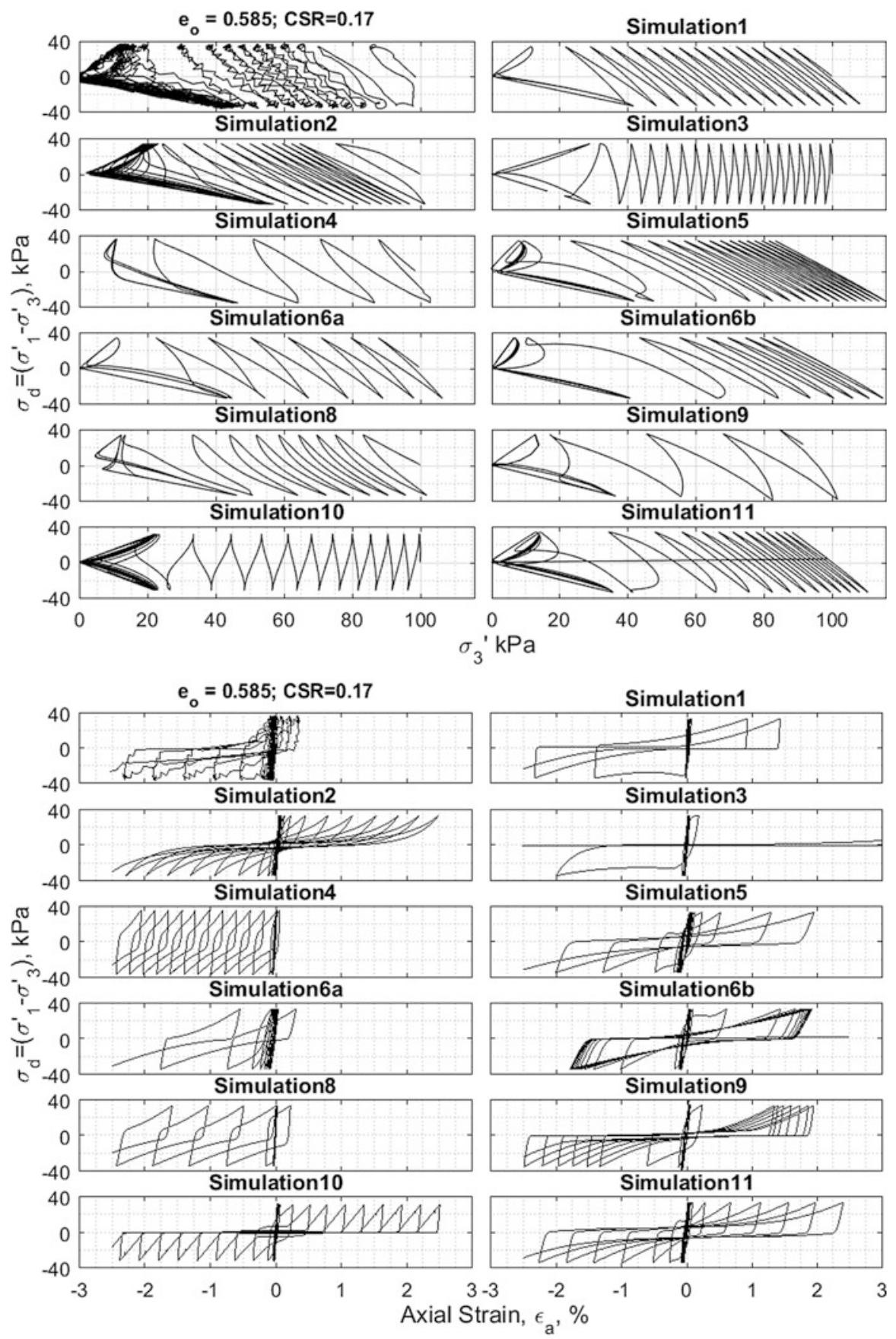

Fig. 9.1 Comparison of the numerical simulations of a cyclic stress-controlled test on Ottawa F-65 sand for $\rho_{\mathrm{d}}=1666 \mathrm{~kg} / \mathrm{m}^{3}\left(e=0.585, D_{\mathrm{r}} \sim 71.5 \%\right), \mathrm{p}_{0}^{\prime}=100 \mathrm{kPa}, \mathrm{CSR}=0.17$ 
The constitutive model and the finite element/difference platform used by each numerical simulation team are also listed in the above table. More detailed information about each constitutive model and the numerical simulation techniques used by each team are provided in separate papers (Wang et al. 2019; Ozutsumi 2019; Fukutake and Kiriyama 2019; Fasano et al. 2019; Chen et al. 2019; Mercado et al. 2017; Qiu and Elgamal 2019; Wada and Ueda 2019; Yang et al. 2019; Tsiaousi et al. 2019; Ichii et al. 2019; Montgomery and Ziotopoulou 2019).

\subsection{Summary of the Element Test Simulations}

The numerical simulation teams were requested to present their simulations of the 23 cyclic triaxial tests on Ottawa F-65 sand (see El Ghoraiby et al. 2017, 2018, 2019). Majority of simulation teams simulated cyclic triaxial tests. The UCD-Auburn (Montgomery and Ziotopoulou 2019) and Fugro West teams (Tsiaousi et al. 2019) who worked with a plane strain constitutive model performed their single-element simulations under plane strain conditions and submitted a number of cyclic biaxial simulations for comparison with the experimental results. Hence, to be consistent in comparison of the numerical simulations, instead of the usual $\mathrm{p}^{\prime}$-q plot for the undrained stress path, the results are plotted in space of deviatoric stress $\left(\sigma_{d}=\sigma_{1}^{\prime}-\sigma_{3}^{\prime}\right)$ versus minor principal effective stress $\left(\sigma_{3}^{\prime}\right)$.

Figures 9.1 and 9.2 show detailed comparison of the simulated stress paths and stress-strain curves with two of the cyclic stress-controlled tests with a cyclic stress ratio, $\mathrm{CSR}=0.17$ and 0.12 , performed on specimens with a density of $1666 \mathrm{~kg} / \mathrm{m}^{3}$ which, for Gs $=2.65,\left(\rho_{\mathrm{d}}\right)_{\max }=1757 \mathrm{~kg} / \mathrm{m}^{3}$, and $\left(\rho_{\mathrm{d}}\right)_{\min }=1491 \mathrm{~kg} / \mathrm{m}^{3}$, corresponds to a void ratio of 0.585 and $D_{\mathrm{r}}=71.5 \%$. The simulations are labelled simulations 1 to 11 . The numbers refer to the order of the simulation teams in the list presented above. The numerical simulation team 6 submitted two different simulations with two different constitutive models but the same finite element platform which are labelled "a" and "b." It is also noted that a few simulation teams did not submit their simulations for all of the requested cyclic stress ratios. Figures 9.3 and 9.4 show similar comparisons of the numerical simulation with the results of cyclic stresscontrolled triaxial tests on denser specimens $\rho_{\mathrm{d}}=1713 \mathrm{~kg} / \mathrm{m}^{3}(e=0.542$, $D_{\mathrm{r}}=87.5 \%$ ) for cyclic stress ratio (CSR) of 0.24 and 0.19 , respectively. Figures 9.5 and 9.6 show the numerical simulations of the stress-controlled cyclic triaxial tests for a much denser specimen $\rho_{\mathrm{d}}=1744 \mathrm{~kg} / \mathrm{m}^{3}\left(e=0.515, D_{\mathrm{r}}=97.5 \%\right)$ for cyclic stress ratio (CSR) of 0.365 and 0.225 , respectively. Numerical simulation team 7 did not submit simulations for these cyclic stress ratios.

Figures 9.1, 9.2, 9.3, 9.4, 9.5, and 9.6 present selected examples of the simulations submitted by the numerical simulation teams. A review of Figs. 9.1, 9.2, 9.3, 9.4, 9.5, and 9.6 reveals the following trends:

1. The numerical simulation 1 showed stress-strain responses that are comparable to those observed in the experiments.

2. While the experimental results show ratcheting of the stress-strain curves toward the negative side (extension) of the axial strain, the stress-strain curves simulated 

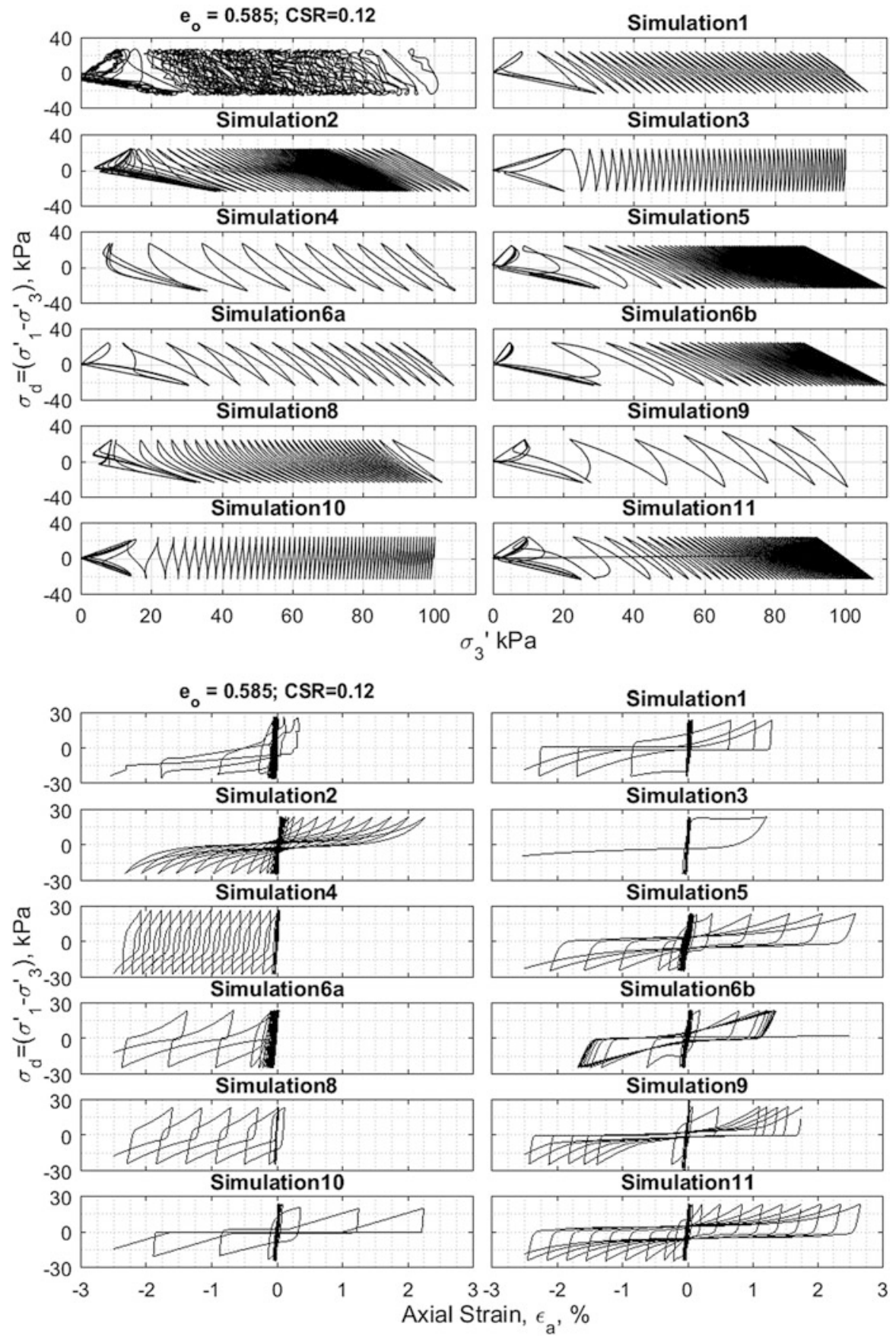

Fig. 9.2 Comparison of the numerical simulations of a cyclic stress-controlled test on Ottawa F-65 sand for $\rho_{\mathrm{d}}=1666 \mathrm{~kg} / \mathrm{m}^{3}\left(e=0.585, D_{\mathrm{r}} \sim 71.5 \%\right), \mathrm{p}^{\prime}{ }_{0}=100 \mathrm{kPa}, \mathrm{CSR}=0.12$ 

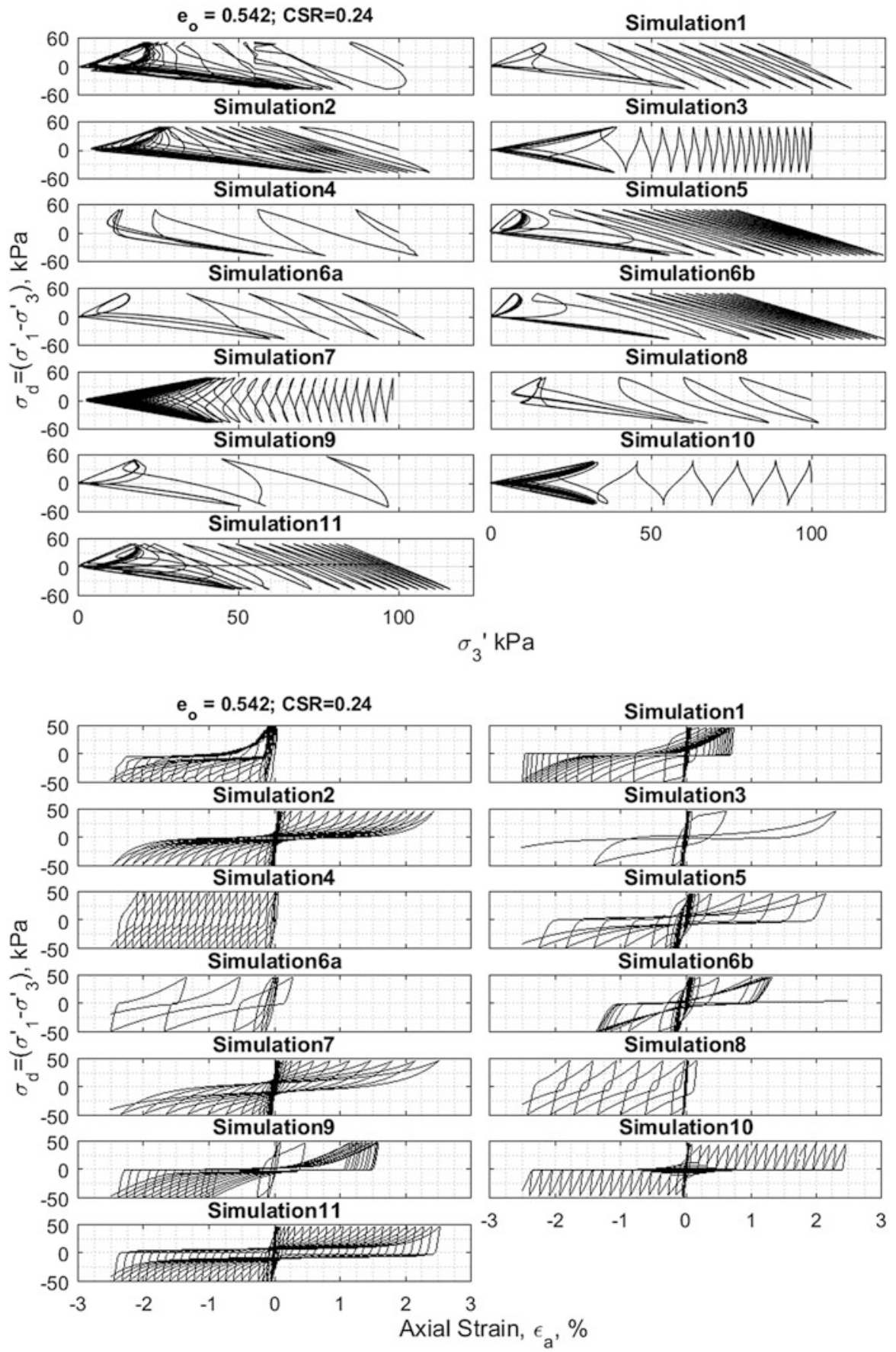

Fig. 9.3 Comparison of the numerical simulations of a cyclic stress-controlled test on Ottawa F-65 sand for $\rho_{\mathrm{d}}=1713 \mathrm{~kg} / \mathrm{m}^{3}\left(e=0.542, D_{\mathrm{r}} \sim 87.5 \%\right), \mathrm{p}_{0}^{\prime}=100 \mathrm{kPa}, \mathrm{CSR}=0.24$ 

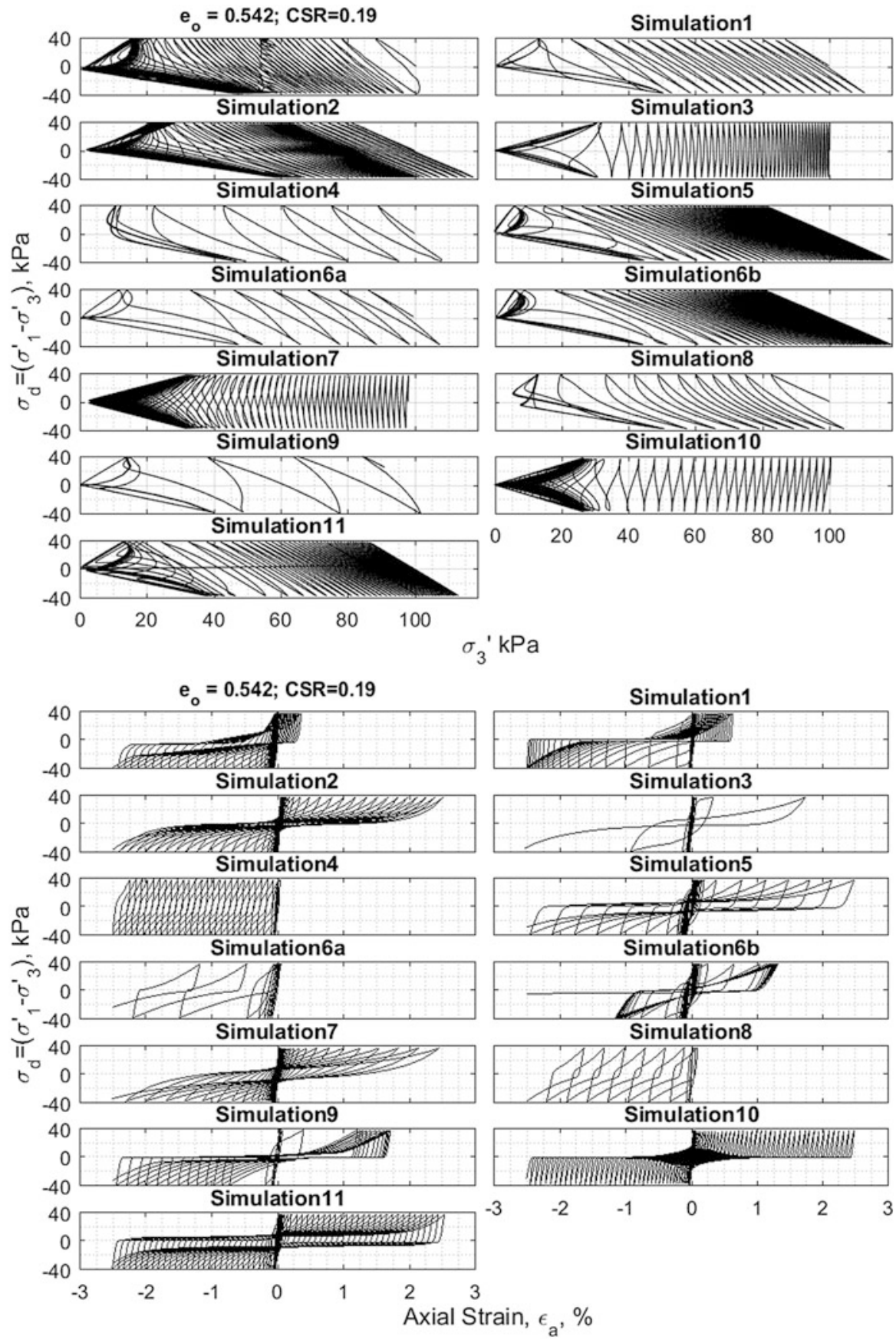

Fig. 9.4 Comparison of the numerical simulations of a cyclic stress-controlled test on Ottawa F-65 sand for $\rho_{\mathrm{d}}=1713 \mathrm{~kg} / \mathrm{m}^{3}\left(e=0.542, D_{\mathrm{r}} \sim 87.5 \%\right), \mathrm{p}_{0}^{\prime}=100 \mathrm{kPa}, \mathrm{CSR}=0.19$ 

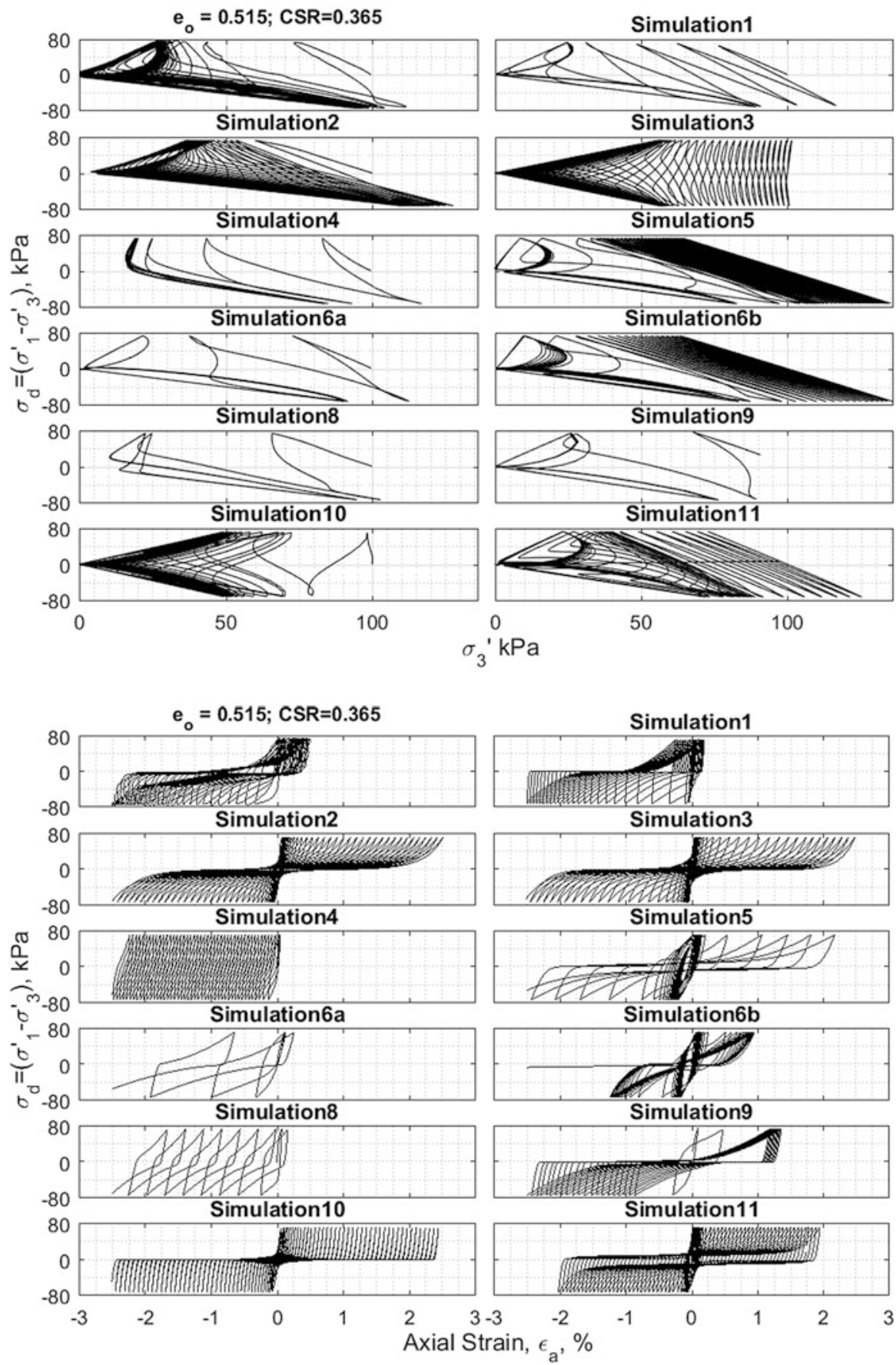

Fig. 9.5 Comparison of the numerical simulations of a cyclic stress-controlled test on Ottawa F-65 sand for $\rho_{\mathrm{d}}=1744 \mathrm{~kg} / \mathrm{m}^{3}\left(e=0.515, D_{\mathrm{r}}=97.5 \%\right), \mathrm{p}_{0}^{\prime}=100 \mathrm{kPa}, \mathrm{CSR}=0.365$ 

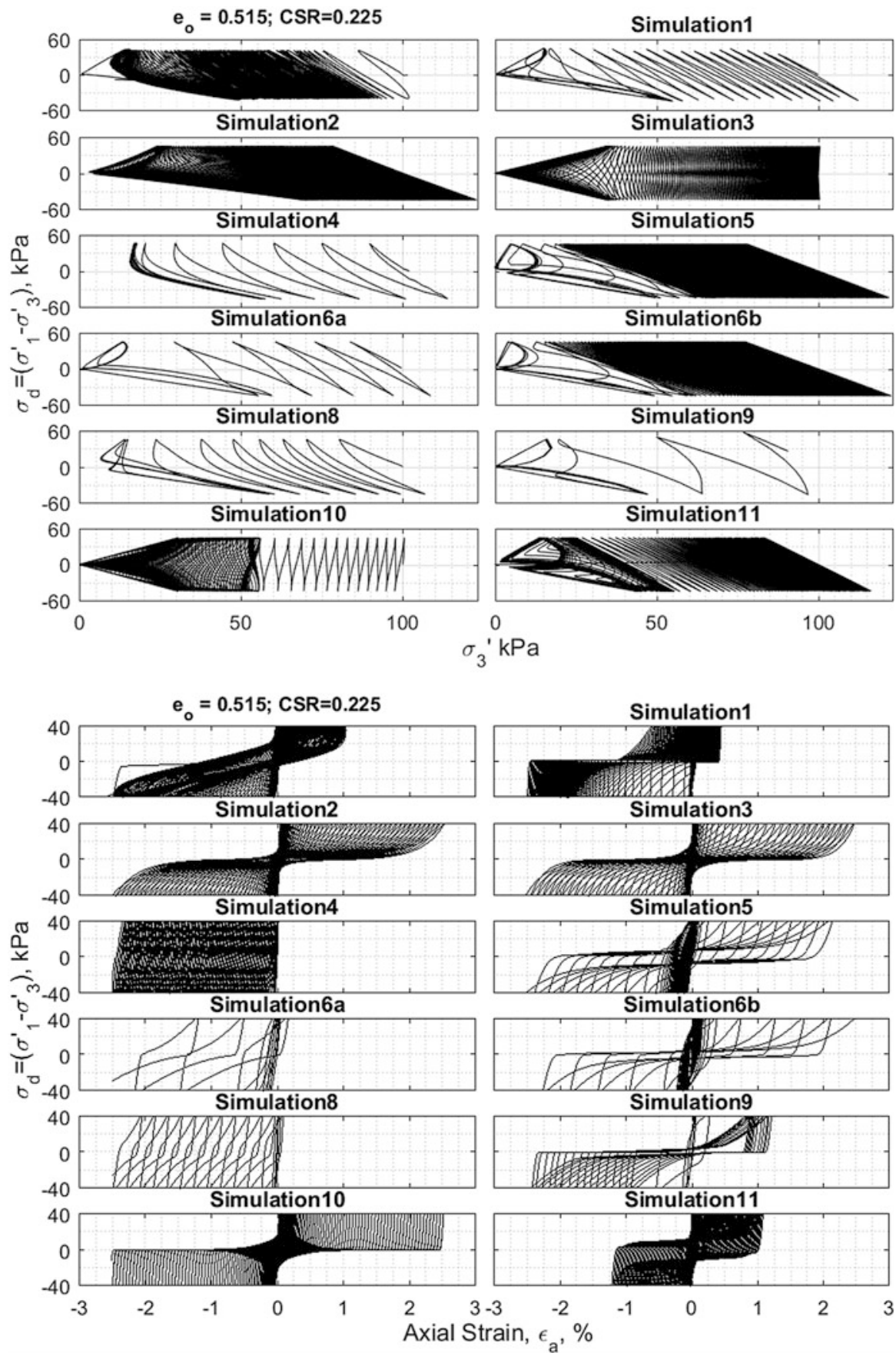

Fig. 9.6 Comparison of the numerical simulations of a cyclic stress-controlled test on Ottawa F-65 sand for $\rho_{\mathrm{d}}=1744 \mathrm{~kg} / \mathrm{m}^{3}\left(e=0.515, D_{\mathrm{r}}=97.5 \%\right), p_{0}^{\prime}=100 \mathrm{kPa}, \mathrm{CSR}=0.225$ 
by the simulation teams $2,3,5,6 \mathrm{~b}, 7,10$, and 11 are more or less symmetric and do not show a visible bias in the ratcheting response.

3. The numerical simulations $6 \mathrm{a}$ show larger ratcheting in the triaxial extension direction compared to the experimental results. Numerical simulations 8 are qualitatively comparable to the numerical simulation of $6 \mathrm{a}$, but show smaller ratcheting and seem to have stabilized at larger effective stresses than those shown in numerical simulation 6 .

4. The stress paths of numerical simulation 4 appear to have stabilized at an effective stresses larger than those observed in the cyclic triaxial experiments.

5. Numerical simulation 9 shows stress-strain responses similar to those observed in the experiments; however, they have reached a larger double amplitude axial strains at a lower number of cycles than those observed in the experiments.

6. In the experiments, the amplitude of the cyclic strains continues to grow with each additional cycle of loading. After some number of cycles, the amplitude of the cyclic strains stops growing in simulations $1,4,6 \mathrm{a}, 6 \mathrm{~b}$, and 8 . The peak amplitude of cyclic strain is significantly underestimated by simulations 4 and 8 .

To gain additional insights into the performance of each model, the experimentally observed and numerically simulated excess pore pressure ratios are plotted against the number of cycles for selected tests (Figs. 9.7, 9.8, and 9.9). All of the models predicted cyclic pore water pressures toward the end of the simulations. It is easy to compare the rate of pore pressure generation in the plots of pore pressure ratio as a function of number of cycles. The rate of pore pressure generation varied significantly from model to model.

\subsection{Liquefaction Strength Curves}

The liquefaction strength curves simulated by each team for the three void ratios of $0.585,0.542$, and $0.515\left(D_{\mathrm{r}}=71.5 \%, 87.5 \%\right.$, and $\left.97.5 \%\right)$ are summarized in Figs. 9.10, 9.11, and 9.12, respectively. The following trends are observed from these curves:

1. For the largest void ratio $(e=0.585)$, numerical simulations 6 a and 9 showed significantly steeper curves than the experimental curves. Numerical simulation 9 showed a reduction in number of cycles for CSRs lower than 0.16 compared to larger CSRs. Numerical simulation 3, while showing a trend consistent with the experimentally observed curve, used different CSRs than those specified in the guidelines for simulations.

2. For the denser specimens with $e=0.542$, simulations $6 \mathrm{a}, 8$, and 9 predicted much steeper curves than the experimental curve. For this void ratio, the simulation 11 showed noticeably larger number of cycles than those observed in the experiments.

3. The quality of the match to the experimentally obtained curve for the densest case $(e=0.515)$ appears to be significantly lower for a large number of models. 
a

$e_{0}=0.585 ; C S R=0.17$
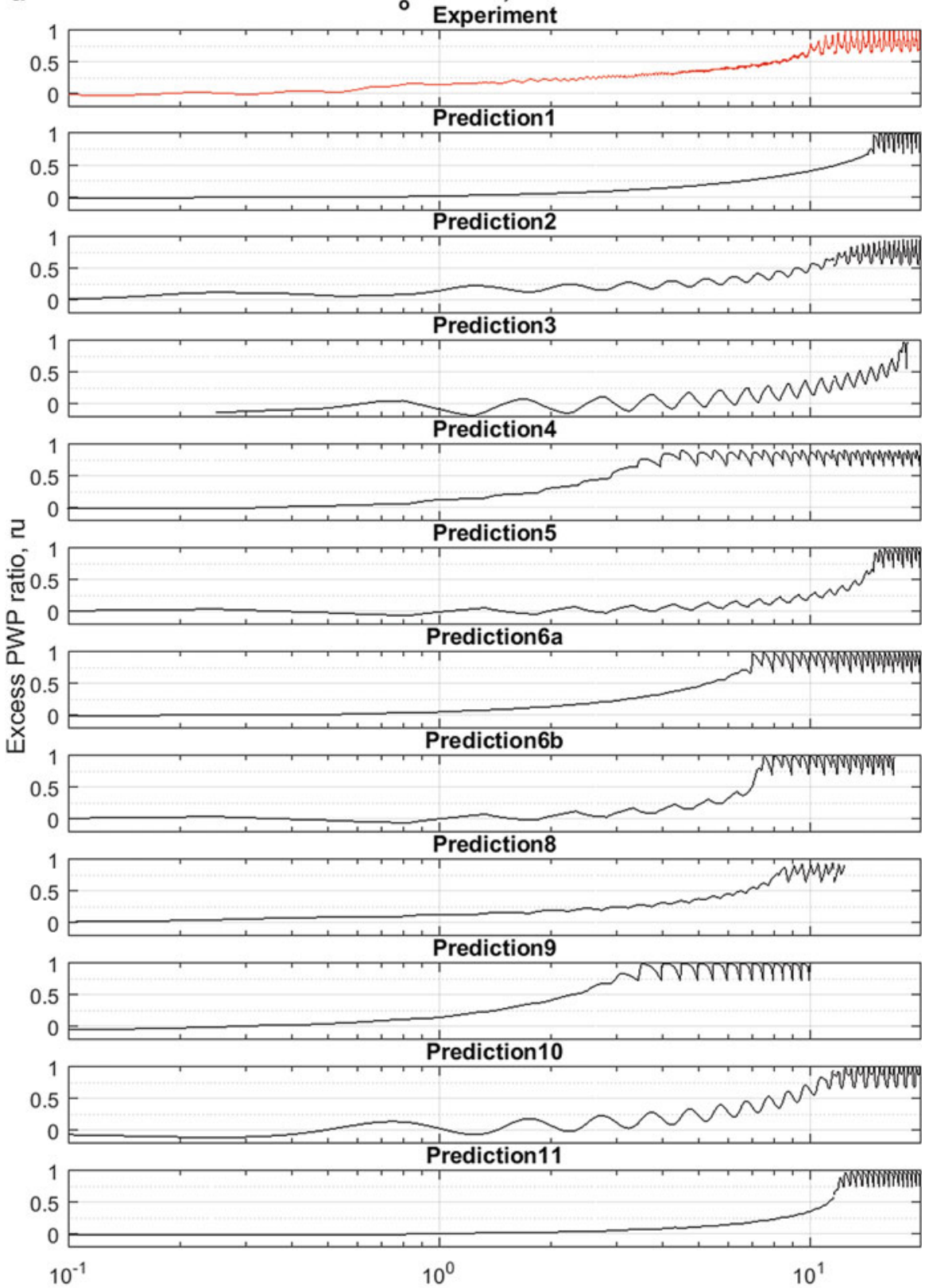

No. of Cycles

Fig. 9.7 (a) Comparisons of the observed versus computed excess pore water pressure ratios with number of cycles for $\rho_{\mathrm{d}}=1666 \mathrm{~kg} / \mathrm{m}^{3}\left(e=0.585, D_{\mathrm{r}} \sim 71.5 \%\right)$ at CSR $=0.17$. (b) Comparisons of the observed versus computed excess pore water pressure ratios with number of cycles for $\rho_{\mathrm{d}}=1666 \mathrm{~kg} / \mathrm{m}^{3}\left(e=0.585, D_{\mathrm{r}} \sim 71.5 \%\right)$ at $\mathrm{CSR}=0.12$ 
b

$e_{0}=0.585 ; C S R=0.12$
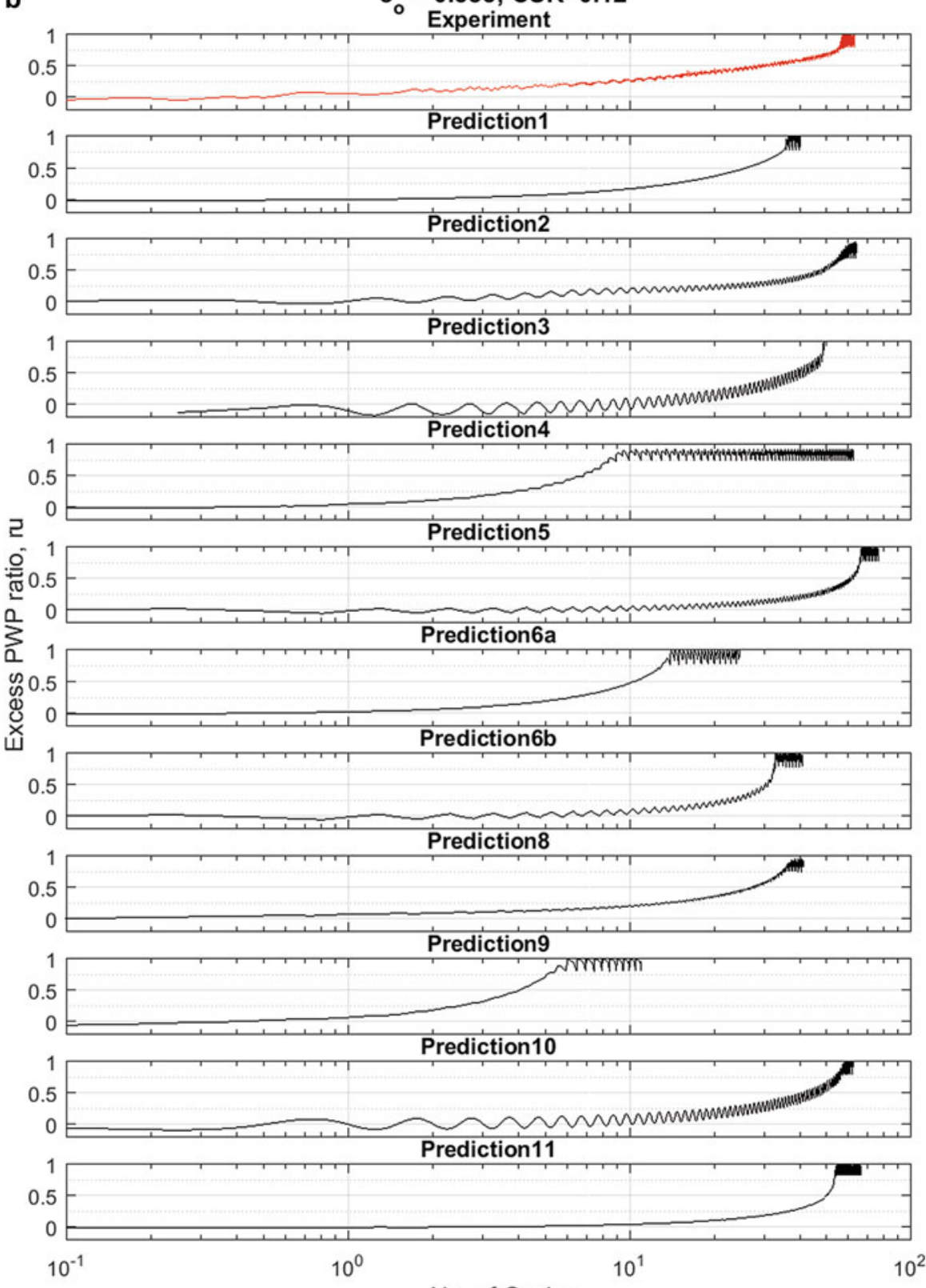

No. of Cycles

Fig. 9.7 (continued) 
a

$e_{0}=0.542 ; C S R=0.24$
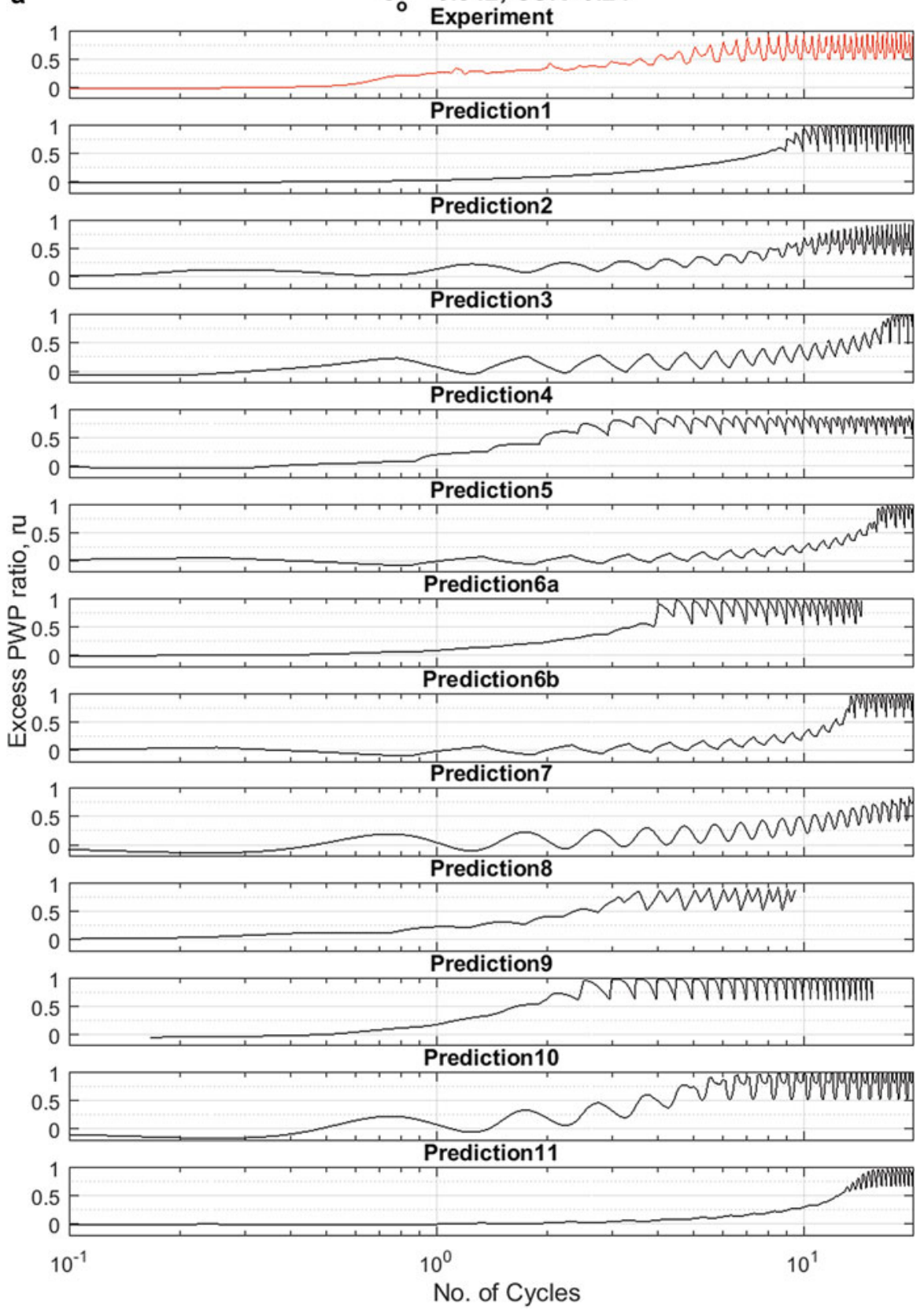

Fig. 9.8 (a) Comparisons of the observed versus computed excess pore water pressure ratios with number of cycles for $\rho_{\mathrm{d}}=1713 \mathrm{~kg} / \mathrm{m}^{3}\left(e=0.542, D_{\mathrm{r}} \sim 87.5 \%\right)$ at CSR $=0.24$. (b) Comparisons of the observed versus computed excess pore water pressure ratios with number of cycles for $\rho_{\mathrm{d}}=1713 \mathrm{~kg} / \mathrm{m}^{3}\left(e=0.542, D_{\mathrm{r}} \sim 87.5 \%\right)$ at $\mathrm{CSR}=0.19$ 
b

$e_{0}=0.542 ; C S R=0.19$
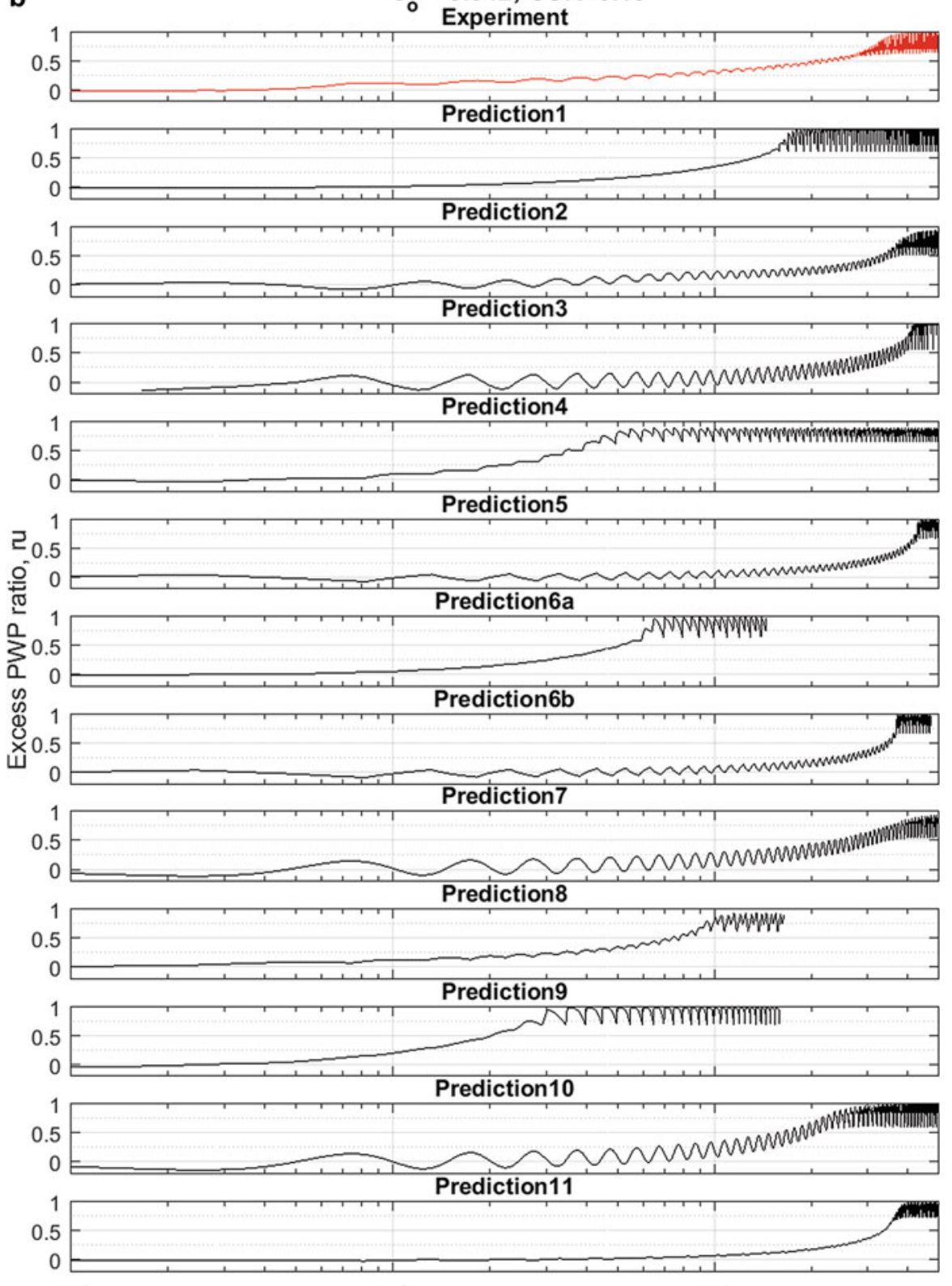

$\begin{array}{lll}10^{-1} & 10^{0} & 10^{1}\end{array}$

No. of Cycles

Fig. 9.8 (continued) 
a $e_{0}=0.515 ; \operatorname{CSR}=0.365$ Experiment
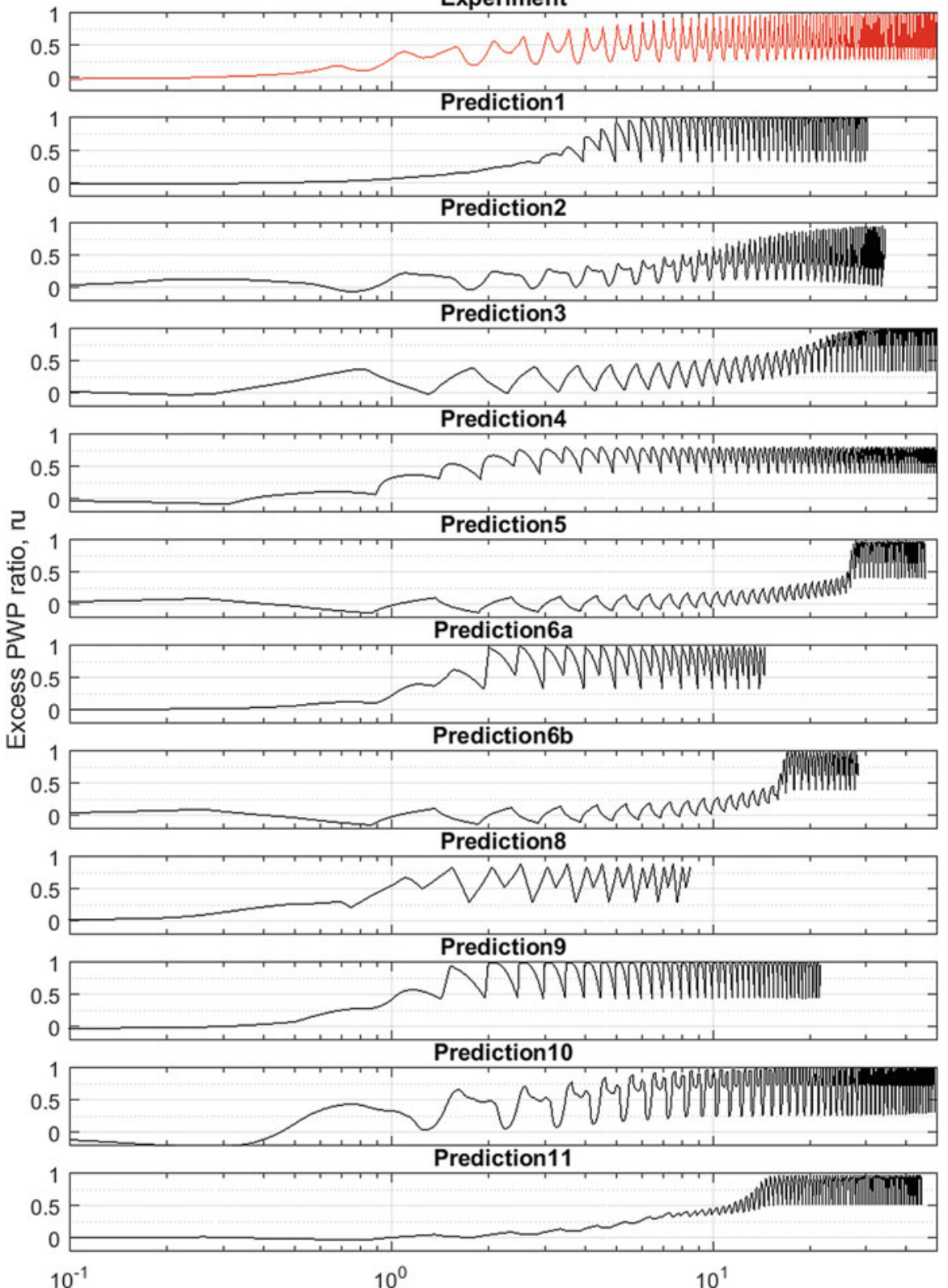

No. of Cycles

Fig. 9.9 (a) Comparisons of the observed versus computed excess pore water pressure ratios with number of cycles. $\rho_{\mathrm{d}}=1744 \mathrm{~kg} / \mathrm{m}^{3}\left(e=0.515, D_{\mathrm{r}} \sim 97.5 \%\right)$ at CSR $=0.365$. (b) Comparisons of the observed versus computed excess pore water pressure ratios with number of cycles. $\rho_{\mathrm{d}}=1744 \mathrm{~kg} /$ $\mathrm{m}^{3}\left(e=0.515, D_{\mathrm{r}} \sim 97.5 \%\right)$ at $\mathrm{CSR}=0.225$ 
b $e_{0}=0.515 ; C S R=0.225$ Experiment

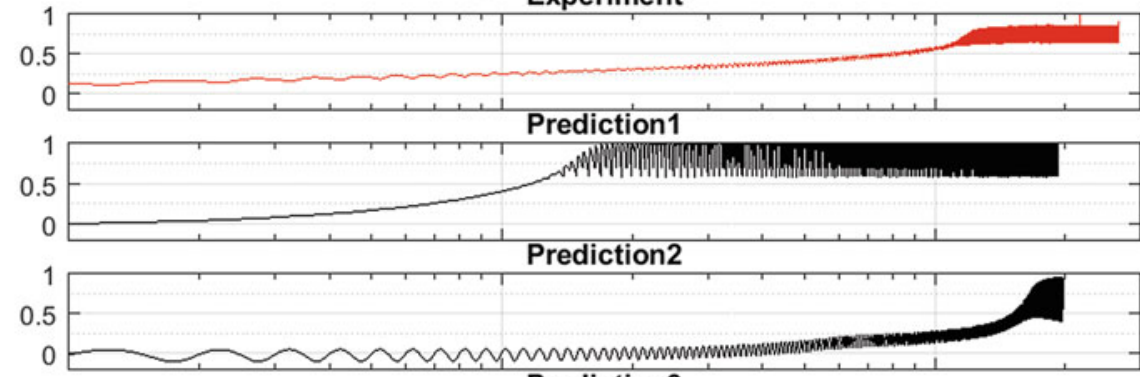

Prediction3

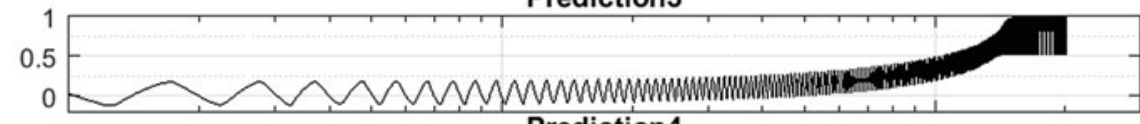

Prediction4
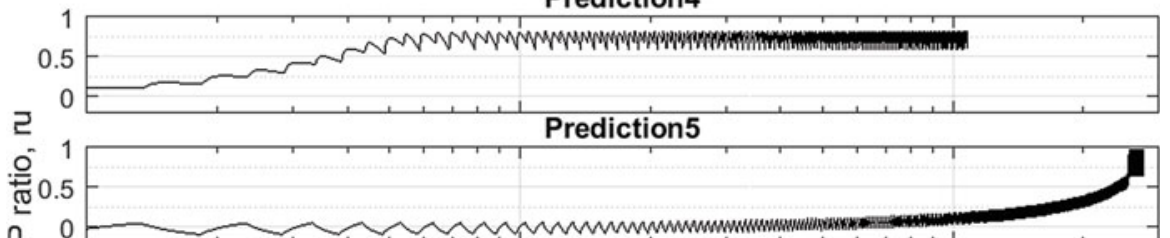

30 Prediction6a
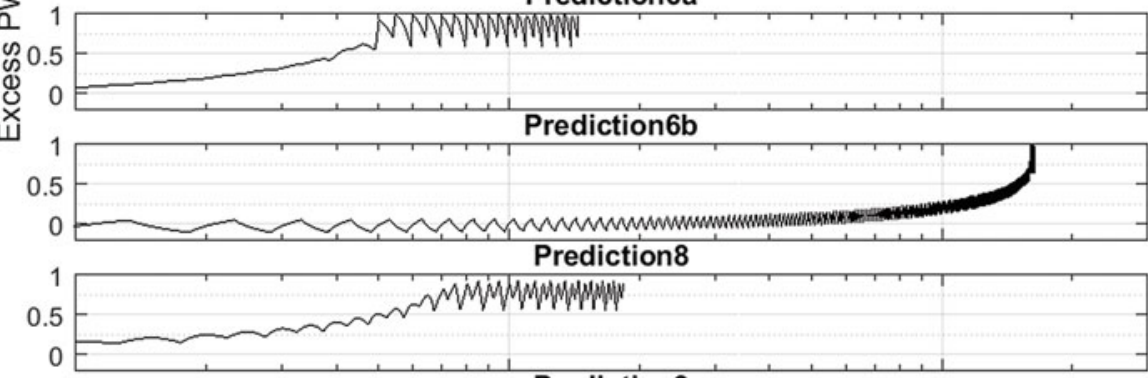

\section{Prediction9}
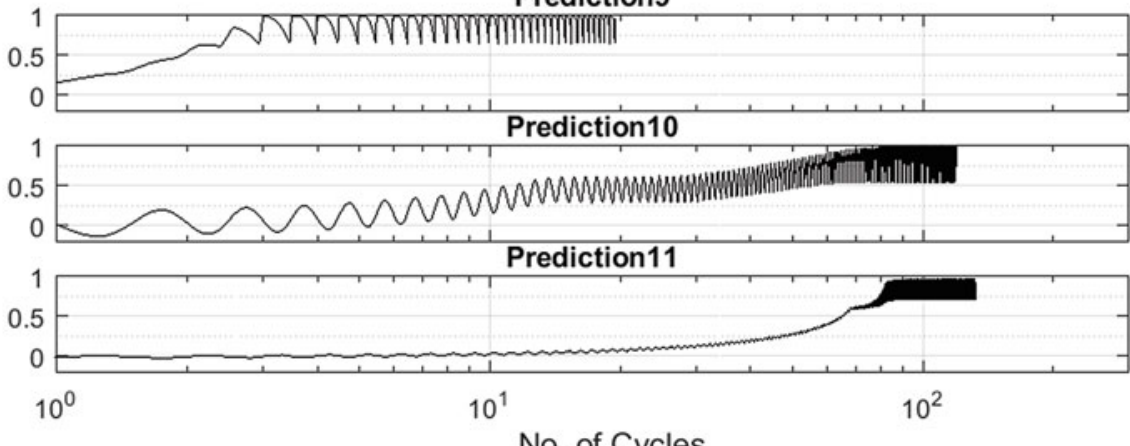

No. of Cycles

Fig. 9.9 (continued) 


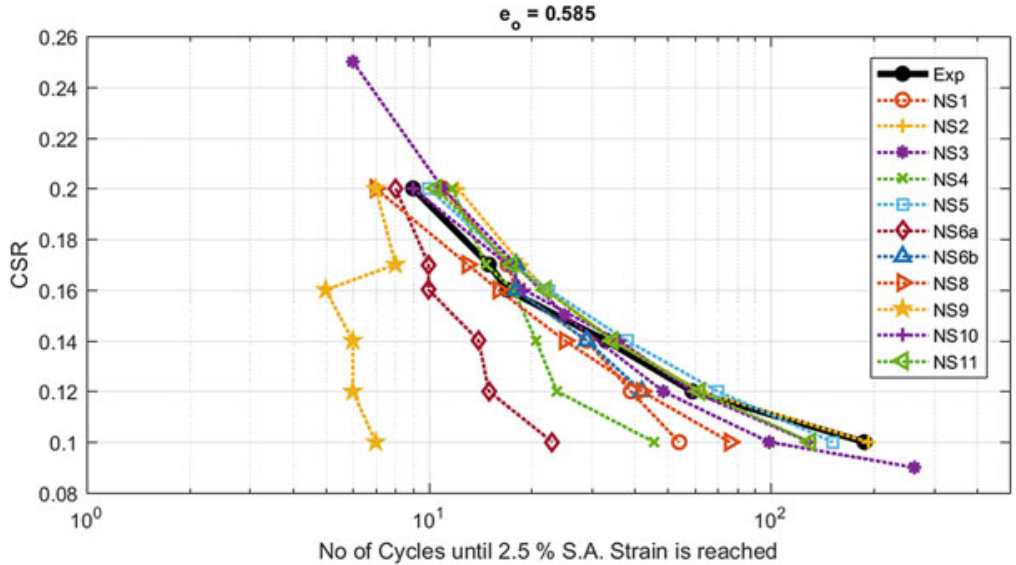

Fig. 9.10 Comparison of the simulated liquefaction strength curves by different numerical simulations teams with the experimental results reported by El Ghoraiby et al. $(2017,2018,2019)$ for $\rho_{\mathrm{d}}=1666 \mathrm{~kg} / \mathrm{m}^{3}\left(e=0.585, D_{\mathrm{r}} \sim 71.5 \%\right)$

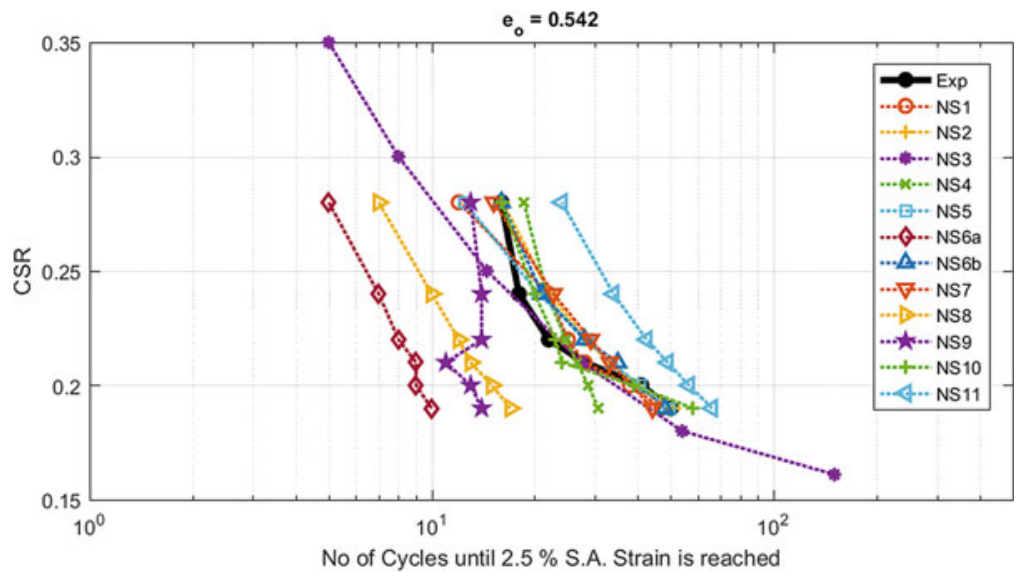

Fig. 9.11 Comparison of the simulated liquefaction strength curves by different numerical simulations teams with the experimental results reported by El Ghoraiby et al. $(2017,2018,2019)$ for $\rho_{\mathrm{d}}=1714 \mathrm{~kg} / \mathrm{m}^{3}\left(e=0.542, D_{\mathrm{r}} \sim 87.5 \%\right)$

\subsection{Conclusions}

This article presented a summary of the numerical simulations of cyclic triaxial tests submitted by various numerical simulation teams as part of the calibration phase of LEAP-2017 project. These simulations show that a significant number of the constitutive models used in these simulations are able to capture the overall trends 


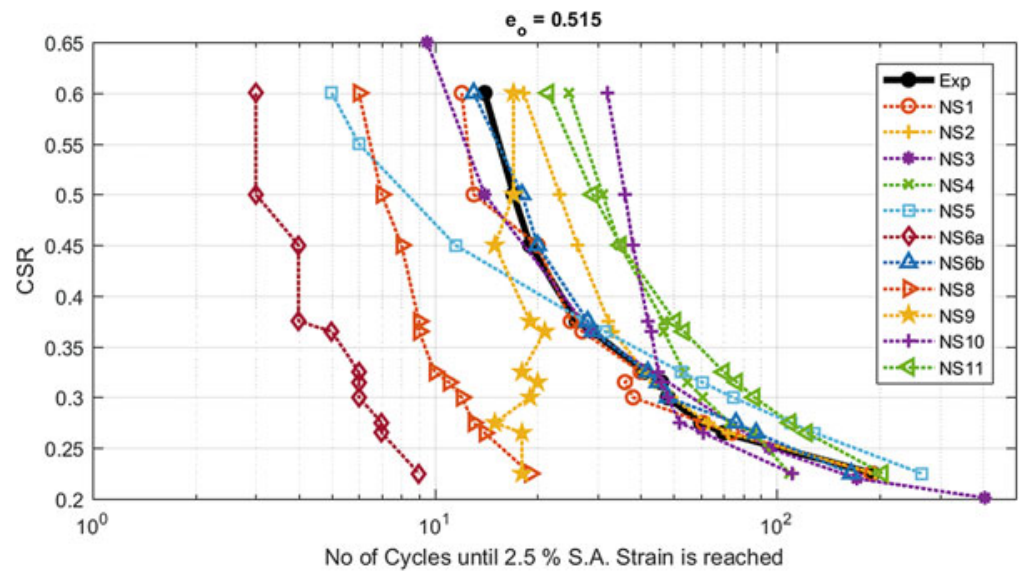

Fig. 9.12 Comparison of the simulated liquefaction strength curves for $\rho_{\mathrm{d}}=1744 \mathrm{~kg} / \mathrm{m}^{3}$ $\left(e=0.515, D_{\mathrm{r}} \sim 97.5 \%\right)$ by different numerical simulations teams with the experimental results reported by El Ghoraiby et al. $(2017,2018,2019)$

of the stress-strain response of Ottawa F-65 sand at medium dense condition $\left(e=0.585, D_{\mathrm{r}} \sim 70 \%\right)$. However, capturing the liquefaction strength curves for all the three selected void ratios appeared to be quite challenging.

Acknowledgments The experimental work and numerical simulations on LEAP-UCD-2017 was supported by different funds depending mainly on the location of the work. The work by the US PIs (Manzari, Kutter, and Zeghal) is funded by National Science Foundation grants: CMMI 1635524, CMMI 1635307, and CMMI 1635040. Partial funding from Caltrans supported the efforts of co-authors Elgamal and Qiu.

\section{References}

Bastidas, A. M. P. (2016). Ottawa F-65 Sand Characterization. PhD Dissertation, University of California, Davis.

Bastidas, A. M. P., Boulanger, R. W., Carey, T., \& DeJong, J. (2017). Ottawa F-65 Sand Data from Ana Maria Parra Bastidas. https://datacenterhub.org/resources/ottawa_f_65.

Chen, L., Ghofrani, A., \& Arduino, P. (2019). Prediction of LEAP-UCD-2017 Centrifuge test results using two advanced plasticity sand models. In B. Kutter et al. (Eds.), Model tests and numerical simulations of liquefaction and lateral spreading: LEAP-UCD-2017. New York: Springer.

El Ghoraiby, M. A., Park, H., \& Manzari, M. T. (2017). LEAP 2017: Soil characterization and element tests for Ottawa F65 sand. Washington, DC: The George Washington University.

El Ghoraiby, M. A., Park, H., \& Manzari, M. (2018). LEAP-2017 GWU Laboratory Tests. DesignSafe-CI, Dataset. https://doi.org/10.17603/DS2210X.

El Ghoraiby, M. A., Park, H., \& Manzari, M. T. (2019). Physical and mechanical properties of Ottawa F65 Sand. In B. Kutter et al. (Eds.), Model tests and numerical simulations of liquefaction and lateral spreading: LEAP-UCD-2017. New York: Springer. 
Fasano, G., Chiaradonna, A., \& Bilotta, E. (2019). LEAP-UCD-2017 Centrifuge test simulation at UNINA. In B. Kutter et al. (Eds.), Model tests and numerical simulations of liquefaction and lateral spreading: LEAP-UCD-2017. New York: Springer.

Fukutake, K., \& Kiriyama, T. (2019). LEAP-2017 Centrifuge test simulation using HiPER. In B. Kutter et al. (Eds.), Model tests and numerical simulations of liquefaction and lateral spreading: LEAP-UCD-2017. New York: Springer.

Ichii, K., Uemura, K., Orai, N., \& Hyodo, J. (2019). Numerical simulation trial by cocktail glass model in FLIP ROSE for LEAP-UCD-2017. In B. Kutter et al. (Eds.), Model tests and numerical simulations of liquefaction and lateral spreading: LEAP-UCD-2017. New York: Springer.

Mercado, V., Fuentes, W. \& Lascarro, C. (2017). LEAP 2017 Simulation Exercise - Phase I: Model Calibration. Calibration report, June 28, DesignSafe.

Montgomery, J., \& Ziotopoulou, K. (2019). Numerical simulations of selected LEAP centrifuge. In B. Kutter et al. (Eds.), Model tests and numerical simulations of liquefaction and lateral spreading: LEAP-UCD-2017. New York: Springer.

Ozutsumi, O. (2019). LEAP-UCD-2017 numerical simulation at Meisosha Corp. In B. Kutter et al. (Eds.), Model tests and numerical simulations of liquefaction and lateral spreading: LEAP$U C D$-2017. New York: Springer.

Qiu, Z., \& Elgamal, A. (2019). Numerical simulations of LEAP dynamic centrifuge model tests for response of liquefiable sloping ground. In B. Kutter et al. (Eds.), Model tests and numerical simulations of liquefaction and lateral spreading: LEAP-UCD-2017. New York: Springer.

Tsiaousi, D., Ugalde, J., \& Travasarou, T. (2019). LEAP-UCD-2017 simulation team Fugro. In B. Kutter et al. (Eds.), Model tests and numerical simulations of liquefaction and lateral spreading: LEAP-UCD-2017. New York: Springer.

Vasko, A. (2015). An investigation into the behavior of Ottawa sand through monotonic and cyclic shear tests. Masters Thesis, The George Washington University.

Vasko, A., El Ghoraiby, M., \& Manzari, M., (2018). LEAP-GWU-2015 Laboratory Tests. DesignSafe-CI, Dataset, https://doi.org/10.17603/DS2TH7Q

Wada, T., \& Ueda, K. (2019). LEAP-UCD-2017 type-B predictions through FLIP at Kyoto University. In B. Kutter et al. (Eds.), Model tests and numerical simulations of liquefaction and lateral spreading: LEAP-UCD-2017. New York: Springer.

Wang, R., Chen, R., \& Zhang, J.-M. (2019). LEAP-UCD-2017 simulations at Tsinghua University. In B. Kutter et al. (Eds.), Model tests and numerical simulations of liquefaction and lateral spreading: LEAP-UCD-2017. New York: Springer.

Yang, M., Barrero, A. R., \& Taiebat, M. (2019). Application of a SANISAND model for numerical simulations of the LEAP 2017 experiments. In B. Kutter et al. (Eds.), Model tests and numerical simulations of liquefaction and lateral spreading: LEAP-UCD-2017. New York: Springer.

Open Access This chapter is licensed under the terms of the Creative Commons Attribution 4.0 International License (http://creativecommons.org/licenses/by/4.0/), which permits use, sharing, adaptation, distribution and reproduction in any medium or format, as long as you give appropriate credit to the original author(s) and the source, provide a link to the Creative Commons licence and indicate if changes were made.

The images or other third party material in this chapter are included in the chapter's Creative Commons licence, unless indicated otherwise in a credit line to the material. If material is not included in the chapter's Creative Commons licence and your intended use is not permitted by statutory regulation or exceeds the permitted use, you will need to obtain permission directly from the copyright holder.

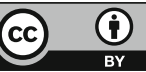

